\title{
The new double energy-velocity spectrometer VERDI
}

\author{
Kaj Jansson ${ }^{1}$, Marc Olivier Frégeau ${ }^{2}$, Ali Al-Adili ${ }^{1}$, Alf Göök ${ }^{3}$, Cecilia Gustavsson ${ }^{1}$, Franz-Josef Hambsch ${ }^{3}$, \\ Stephan Oberstedt ${ }^{3, \text { a }}$, and Stephan Pomp ${ }^{1}$ \\ 1 Department of Physics and Astronomy, Uppsala University, Box 516, 75120 Uppsala, Sweden \\ 2 GANIL CEA/DRF-CNRS/IN2P3, Bd. Henri Becquerel, BP. 55027, 14076 Caen Cedex 05, France \\ 3 EC-JRC-Dir. G-Unit G.2, Retieseweg 111, 2440 Geel, Belgium
}

\begin{abstract}
VERDI (VElocity foR Direct particle Identification) is a fission-fragment spectrometer recently put into operation at JRC-Geel. It allows measuring the kinetic energy and velocity of both fission fragments simultaneously. The velocity provides information about the pre-neutron mass of each fission fragment when isotropic prompt-neutron emission from the fragments is assumed. The kinetic energy, in combination with the velocity, provides the post-neutron mass. From the difference between pre- and post-neutron masses, the number of neutrons emitted by each fragment can be determined. Multiplicity as a function of fragment mass and total kinetic energy is one important ingredient, essential for understanding the sharing of excitation energy between fission fragments at scission, and may be used to benchmark nuclear de-excitation models. The VERDI spectrometer design is a compromise between geometrical efficiency and mass resolution. The spectrometer consists of an electron detector located close to the target and two arrays of silicon detectors, each located $50 \mathrm{~cm}$ away from the target. In the present configuration pre-neutron and post-neutron mass distributions are in good agreement with reference data were obtained. Our latest measurements performed with spontaneously fissioning ${ }^{252} \mathrm{Cf}$ is presented along with the developed calibration procedure to obtain pulse height defect and plasma delay time corrections.
\end{abstract}

\section{Introduction}

In the fission process fragments with a very large distribution in mass, kinetic energy and excitation energy are created. Good knowledge of the yields for each isotope produced is important for applications like nuclear reactors and radioactive waste transmutation. With the advent of accelerator driven system, fission yield dependence to excitation energy of the fissioning nuclei will become especially important [1].

How the mass is shared between the nascent fragments is subject to many theoretical models, but none has yet managed to produce a predictive description of good accuracy [2-5] and very few have even started to consider the influence of excitation energy [6]. The distribution of excitation energy between the fragments $[7,8]$ is also a very disputed question [9-12]. In order to guide theory, it is important to provide theorists and evaluators with experimental data containing as many correlations as possible between the different observables, such as fragments mass, charge, kinetic energy and emitted particle multiplicities. Neutron multiplicities are directly related to how the excitation energy is shared at scission.

VERDI provides the fission fragments' masses, kinetic energies and neutron multiplicities using the double energy and double velocity $(2 E-2 v)$ method. In its purest form this method is valid for excitation energies up to the compound nucleus's neutron emission threshold. The main assumption of the $2 E-2 v$ method is that the prompt neutron emission is isotropic. It means that the average velocity is

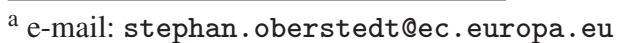

conserved and calculations of both pre- and post-neutron masses can be made through

$$
\begin{gathered}
M_{1}^{\text {pre }}=\frac{v_{2}}{v_{1}+v_{2}} M_{\mathrm{Cf}} \\
M_{1}^{\text {post }}=\sqrt{\frac{2 E_{1}}{v_{1}^{2}}}
\end{gathered}
$$

where $M_{\mathrm{Cf}}$ would be substituted by the mass of the appropriate compound nucleus if not spontaneous fission of ${ }^{252} \mathrm{Cf}$, as in this case, is studied.

\section{VERDI setup and upgrade}

The energy measurement is achieved by 32 silicon detectors located at about $50 \mathrm{~cm}$ from the target. They are arranged in two arrays, each housing 16 detectors on their respective side of the target. These detectors measure the kinetic energy and the arrival (stop) time of the incoming fission products. Emission (start) time of the fragments is determined by micro channel plate (MCP) detectors. The electrons sputtered from the surface of the actinide sample are deflected onto the MCPs by electrostatic mirrors. A complete description of the setup using one MCP and the same silicon arrays as in this work was made in Ref. [13].

Significant difficulties were observed arising from the use of a single MCP, since electrons are mainly detected from one side of the sample. $\alpha$-particles emitted away from the MCP will mainly lead to electron emission in that same direction and would therefore often not be detected by the single MCP. Nonetheless, it is necessary to be 
able to detect $\alpha$-particles in both directions for calibration purposes. In order to correct for the limitations inherent to the use of a single MCP detector, a second MCP has been installed.

After this upgrade we concluded that the efficiency of coincident events (both fission products detected in opposing silicon detectors) compared to non-coincident events had improved from $\sim 32 \%$ to $\sim 44 \%$. The latter efficiency is close to what is expected after effects of particle losses in acceleration grids and non co-linearity has been taken into account. In addition, the efficiencies on both sides are now equal, whereas a few per cent difference was seen before.

\section{Calibration}

The energy calibration mainly follows the procedure of Schmitt et al. [14] but, unlike earlier [13], it also includes information from the $\alpha$-peak. The latter is required to improve the calibration for alpha and ternary particles which could be studied with VERDI in the future.

A correction for the fragment's energy loss in the backing is also made. This correction is applied for both sides of the target since the californium atoms have diffused into the $250 \mathrm{~nm}$ nickel backing. Since the degree of diffusion is not well known, this introduces the diffusion depth as another parameter affecting the calibration.

We are developing an iterative procedure to improve upon the Schmitt calibration since it was found that the energy spectrum of Schmitt is higher than other, newer data [15], and that it also in our analysis led to a too high value of the average pre-neutron total kinetic energy, $\left\langle\mathrm{TKE}_{\mathrm{pre}}\right\rangle$.

\subsection{Plasma delay time}

The time-of-flight calibration was corrected for plasma delay time (PDT) according to the same procedure as outlined in Eqs. ((2)-(6)) in Ref. [13]. Using known neutron multiplicities the plasma-delay-time (PDT) correction was computed event-by-event as the difference between the measured velocity and the one derived from the measured energy. Due to the mass dependence of the energy calibration and the neutron multiplicity, the procedure was run iteratively until the obtained masses converged.

In Fig. 1 it is shown how the PDT corrected velocity scales linearly with the measured velocity. The corrected velocity can therefore be calculated by a linear function once the appropriate linear relationship has been found. Only the linear function mapping measured velocity to the calculated one is used in the final analysis.

\subsection{Improved energy calibration}

The procedure is iterative and makes subsequent corrections to the energy calibration. It scales the velocities to match the literature value of $\left\langle\mathrm{TKE}_{\text {pre }}\right\rangle=184.1 \mathrm{MeV}$ [16]. Since the pre-neutron masses were already found to be in agreement with previous data, both fragment velocities must be scaled by the same constant in order to preserve them Eq. (1). By again utilising known neutron multiplicities together with the corrected velocities, the calculated pre- and post-neutron quantities reproduce the

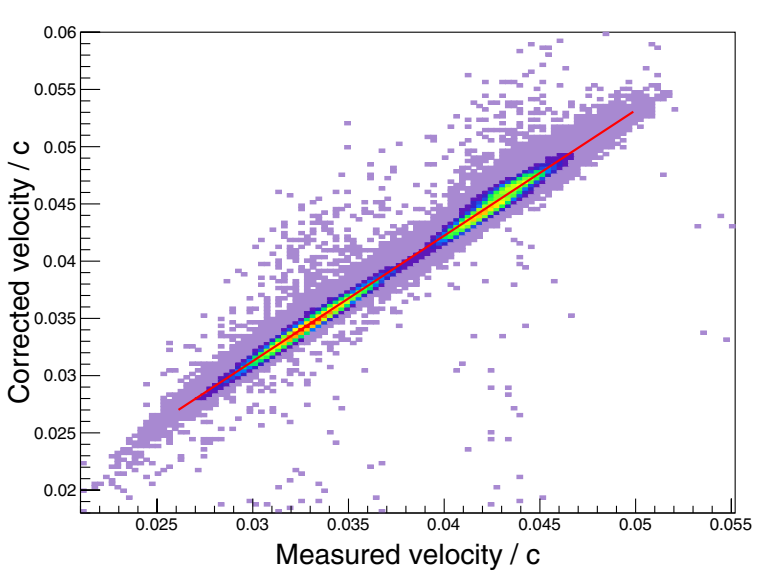

Figure 1. The PDT correction to the velocity scales linearly with the velocity. The line represents a linear fit to the data.

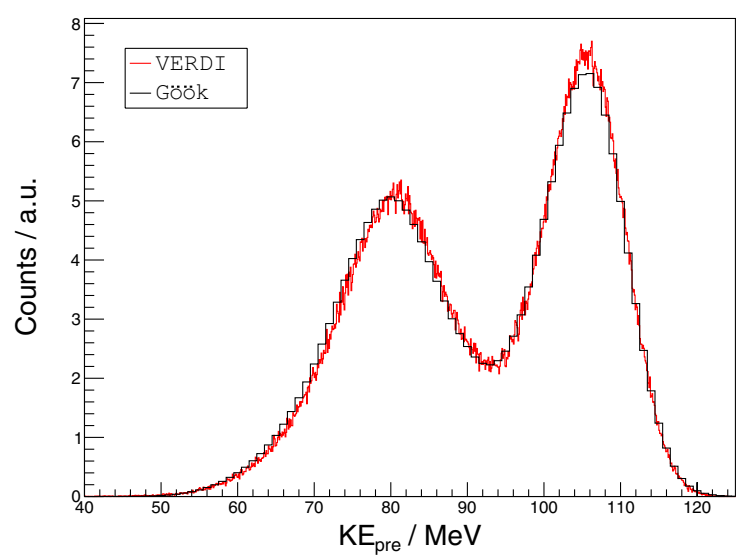

Figure 2. Pre-neutron emission fission fragment kinetic energy measured by VERDI compared to data from Ref. [15].

literature data. Since we get both $M_{\text {post }}$ and $E_{\text {post }}$ on an event-by-event basis we can use them to deduce new values of the four Schmitt parameters [14].

With improved energy calibration the PDT correction changes and $\left\langle\mathrm{TKE}_{\mathrm{pre}}\right\rangle$ approaches the literature value without any scaling. Only the new values of the Schmitt parameters are used in the final analysis. Although this is ongoing work and the method still needs to be refined, some promising results have been obtained in the cases where the recalibration method works well.

\section{Results}

We compare our results to the data from Göök et al. [15] taken using a ionisation chamber, since that allow us to compare all the correlated quantities to a single experiment. Our improved energy calibration does not work equally well for all detector pairs, so we show results from a detector pair where it happens to work well. The energy and mass distributions have been symmetrised as VERDI at the moment has an instrumental asymmetry between the different sides of about $10 \%$ when it comes to detection efficiency of light and heavy fragments, respectively.

Our pre-neutron energy distribution (Fig. 2) is in excellent agreement with the data from Ref. [15], and so is the heavy fragment peak (low energy) of the post-neutron energies (Fig. 3). However, some discrepancies are present 


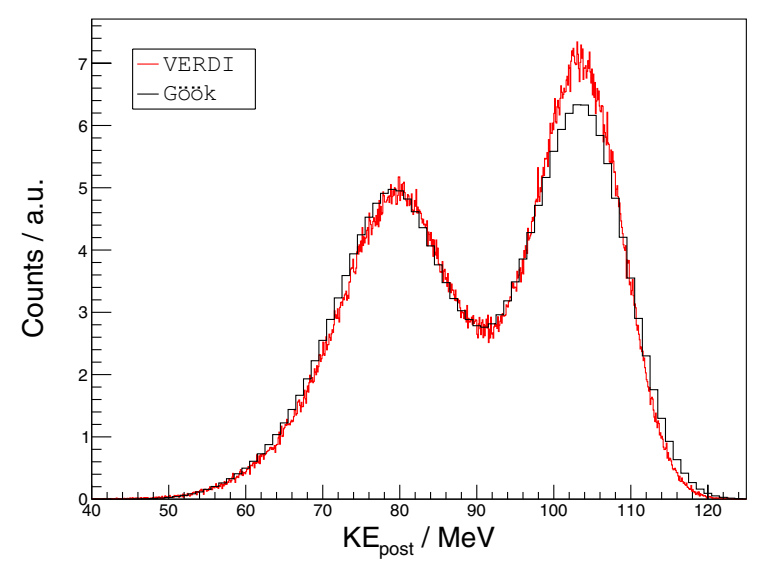

Figure 3. Post-neutron emission fission fragment kinetic energy measured by VERDI compared to data from Ref. [15].

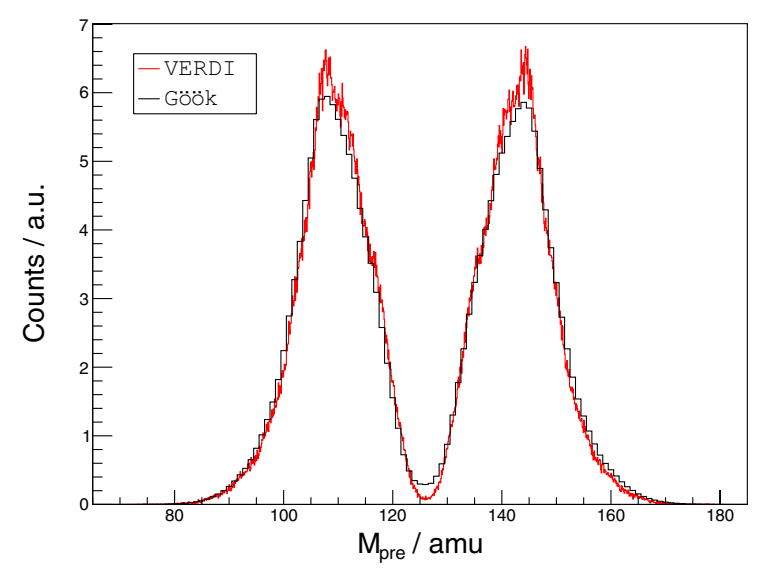

Figure 4. Pre-neutron emission fission fragment mass measured by VERDI compared to data from Ref. [15].

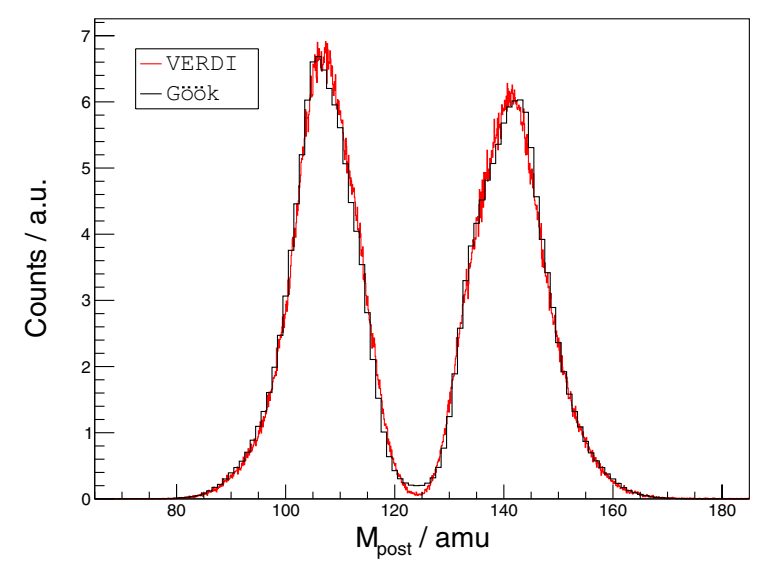

Figure 5. Post-neutron emission fission fragment mass measured by VERDI compared to data from Ref. [15].

in the post-neutron light fragment peak (high energy). Both pre-neutron (Fig. 4) and post-neutron (Fig. 5) masses are in good agreement with Ref. [15], and showing a slightly higher peak-to-valley ratio indicating higher resolution. The general good agreement validate our PDT correction as well as recalibration procedure.

Since both pre- and post-neutron masses are available we can easily calculate the average neutron multiplicity $\bar{v}(A)$. In Fig. $6 \bar{v}$ as a function of $M_{\text {pre }}$ is shown. We can see discrepancies mainly around the symmetry region where our data indicate a negative neutron multiplicity

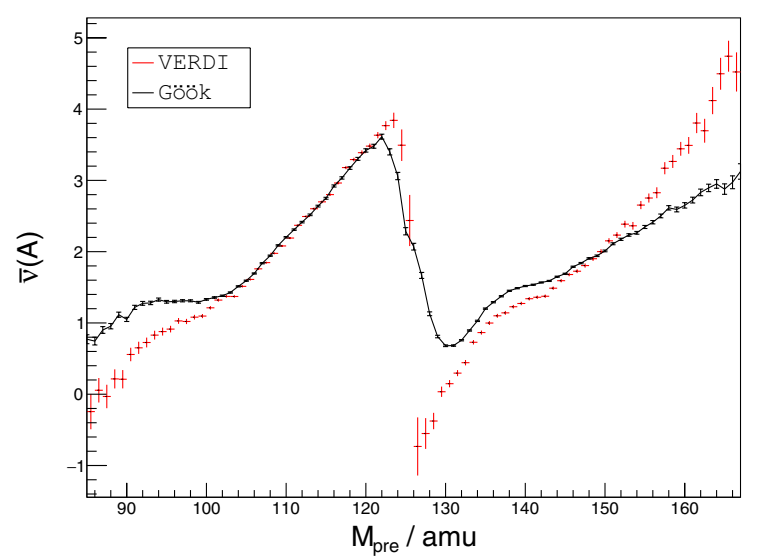

Figure 6. Neutron multiplicity measured by VERDI compared to data from Ref. [15].

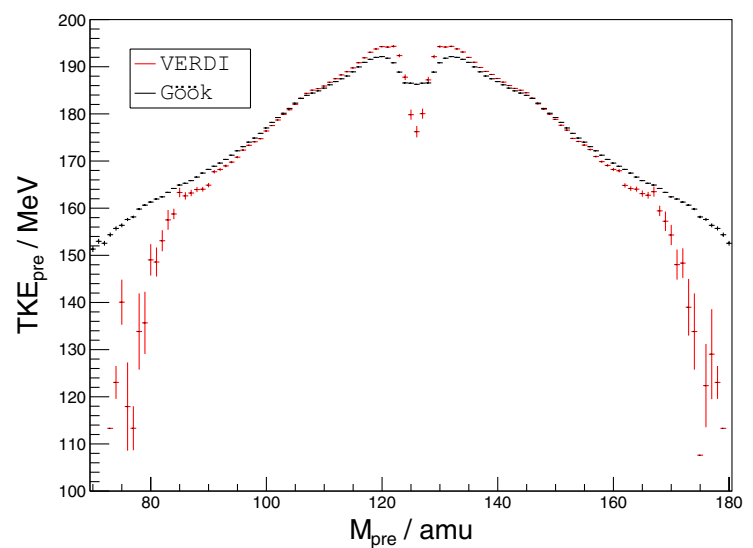

Figure 7. Pre-neutron TKE versus mass measured by VERDI and compared to data from Ref. [15].

which is unphysical. No tweaking, e.g., by using a known average neutron multiplicity for ${ }^{252} \mathrm{Cf}$ has been made, and still a rather good agreement with the data in Ref. [15] is achieved except for the lowest $(A<100)$ and highest $(A>155)$ masses. The total $\bar{v}$ was 3.62 which is within $4 \%$ of the recommended value of $3.759(48)$ for ${ }^{252} \mathrm{Cf}$ [17].

The TKE as a function of $M_{\text {pre }}$ also agrees well with Ref. [15] (Fig. 7) except for masses far from symmetry, and the average TKE before neutron emission, $\left\langle\mathrm{TKE}_{\mathrm{pre}}\right\rangle=$ $184.8 \mathrm{MeV}$, is not too far off from the $184.1(13) \mathrm{MeV}$ recommended value [16] also used by Göök et al. [15] for calibration. The excellent timing properties of the MCP detectors allow for a higher mass resolution as one clearly sees in the symmetry region. Similarly VERDI measures a slightly higher $\mathrm{TKE}_{\text {post }}$ (Fig. 8) with, $\left\langle\mathrm{TKE}_{\text {post }}\right\rangle=$ 182.1 MeV compared to 181.4(13) MeV from Ref. [15].

\section{Conclusion and outlook}

VERDI has now two complete arms with fully independent capabilities, thanks to the second electron mirror and MCP detector. A new calibration procedure is developed which, for the detector pairs where it works well, have successfully produced pre- and post-neutron mass and energy spectra agreeing well with other data. The neutron multiplicity shows some problems around the symmetry region which could be due to a limited $M_{\text {post }}$ resolution but also due to the californium sample used. The deposited californium is assumed to have diffused into the nickel 


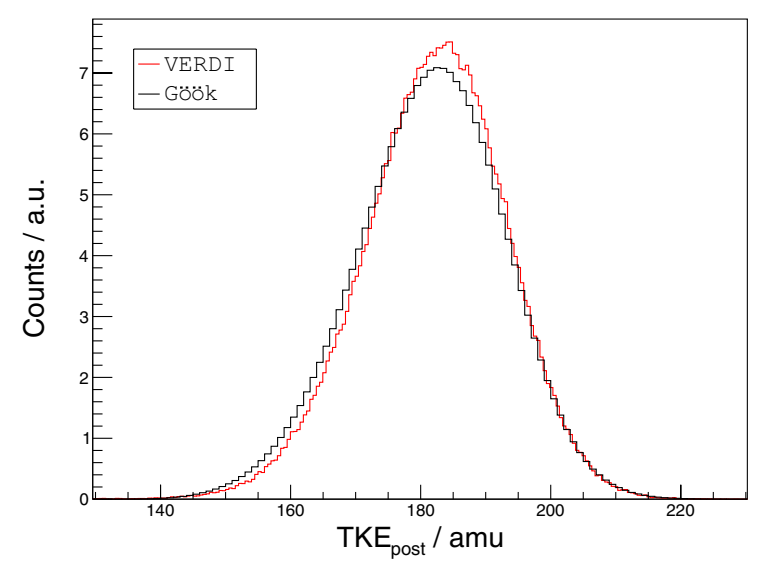

Figure 8. Post-neutron TKE measured by VERDI and compared to data from Ref. [15].

backing and the analysis is affected by how far we assume that the diffusion process has gone. The same sample has been used in a $2 E$ measurement with an ionisation chamber, and problems with the angular distribution were encountered for the backing side (A. Al-Adili, private communication). In future measurements a better sample would be preferred.

Although not shown in Fig. 6, since the data has been symmetrised, the two different arms of VERDI gives very similar and consistent neutron multiplicity indicating that we are handling the asymmetry due to the diffusion in the sample quite well.

In order to measure neutron induced rather that spontaneous fission the mid section of VERDI needs to be refurbished to accommodate a neutron beam. A more flexible placement of the mirror arrangement would certainly be convenient in order to ensure isochronous arrival of the electrons on the MCP [18], something that is not fully supported today due to geometrical constraints. Even when our method is fully developed, the calibration to ${ }^{252} \mathrm{Cf}$ would be necessary to perform whenever the experimental conditions change. With a new mid section a measurement of the thermal fission of ${ }^{235} \mathrm{U}$ would provide a final validation to our method and calibration procedures. After that more exotic nuclei could be explored and high quality correlated data could be supplied to the community.

We sincerely thank W. Geerts, T. Gamboni and M. Vidali for their technical support. K.J. thanks the CHANDA project for its financial support and M.O.F. thanks the EC for awarding him a fellowship at the JRC-GEEL.

\section{References}

[1] Fission Product Yield Data for the Transmutation of Minor Actinide Nuclear Waste, IAEA, Vienna, 2008, p. 341

[2] D. Regnier et al., Phys. Rev. C 93, 054611 (2016)

[3] J. Sadhukhan et al., Phys. Rev. C 93, 011304(R) (2016)

[4] P. Möller \& T. Ichikawa, Eur. Phys. J. A 51, 173 (2015)

[5] Y. Aritomo et al., Phys. Rev. C 88, 044614 (2013)

[6] K.H. Schmidt et al., Nucl. Data. Sheets 131, 107 (2016)

[7] R. Müller et al., Phys. Rev. C 29, 885 (1984)

[8] A. Naqvi et al., Phys. Rev. C 34, 218 (1986)

[9] O. Litaize et al., Phys. Rev. C 82, 054616 (2010)

[10] K.H. Schmidt et al., Phys. Rev. Let. 104, 212501 (2010)

[11] P. Talou et al., Phys. Rev. C 83, 064612 (2011)

[12] T. Kawano et al., J. Nucl. Sci. Technol. 5, 462 (2010)

[13] M.O. Frégeau et al., Nucl. Instrum. Meth. A 817, 35 (2016)

[14] H.W. Schmitt et al., Phys. Rev. 137, B837 (1965)

[15] A. Göök et al., Phys. Rev. C 90, 064611 (2014)

[16] C. Wagemans The Nuclear fission process (CRC Press Inc., Boca Raton Florida, 1991), p. 323

[17] M. Chadwick et al., Nucl. Data Sheets 112, 2887 (2011)

[18] K. Kosev et al., Nucl. Instrum. Meth. A 594, 178-183 (2008) 